\title{
SECUENCIA DE ENSEÑANZA SOBRE EL ELECTROMAGNETISMO
}

\author{
MENESES VILLAGRÁ, J. A. ${ }^{1}$ y CABALLERO SAFELICES, M.C. ${ }^{2}$ \\ ${ }_{1}^{1}$ EU del Profesorado de $\mathrm{EGB}$ de Burgos, Villadiego, s/n 09001 Burgos. \\ 2 Colegio Universitario de Burgos, Sección de Químicas. Av. de Valladolid, s/n 09002 Burgos.
}

\section{SUMMARY}

In orden to plan the teaching sequence of any physical topic, we consider essential to bear in mind three factors, at least: the scientific problems that arise from the subjects to be studied, the conceptions of the students to whom the teaching is addressed, and above all, the strategies and methodological process to be taken. This paper presents the didactic model, based on a constructivist approach, we use, the university students' conceptions about electromagnetism, the conceptual map where we make explicit our points of view of the concepts we work on, and the group activitics we have designed in order to promote the conceptual, methodological and attitudinal change.

\section{INTRODUCCIÓN}

La mayoría de los modelos didácticos renovadores (Batner 1976, Karplus 1977, Erickson 1979, Nussbaum y Novick 1982, Renner 1982, Rovell y Dawson 1983. Needham 1987) que se utilizan hoy día en la enseñanza de las ciencias aportan enfoques, secuencias de instrucción y estrategias didácticas diferentes. En todos, la instrucción parte de las ideas y creencias de los alumnos.

Por este motivo, cn los últimos años, una de las líneas de: investigación sobre la enseñanza/aprendizaje de las ciencias se ha centrado en el estudio de las ideas de los alumnos acerca de determinados tópicos científicos y de las causas que los originan. Algunas conclusiones (Osborne y Wittrock 1983) constatan que los alumnos poseen concepciones resistentes al cambio. Además constituyen en muchos casos un esquema conceptual coherente y con amplio poder explicativo. Estas concepciones interfieren en el aprendizaje de las ciencias ya que, en general, no son congruentes con los conceplos, las leyes y las teorías científicas, siendo responsable, en parte, de la dificultad cue encuentran los alumnos en estas asignaturas y de su bajo rendimiento comparado con otras áreas.

Si bien prolifcran los estudios relacionados con las ideas y esquemas conceptuales de los alumnos en los difercti- tes niveles educativos (Tiberghien y Delacote 1976, Shipstone 1985, Joshua 1984, Psillos et al. 1988, Sebastià 1993), no ocurre lo mismo con los trabajos encaminados hacia la elaboración, puesta en práctica y evaluación de propuestas didácticas concretas.

Con objeto de expandir esta última línea de investigación, presentamos a nivel universitario, nuestra propuesta diđáctica para ta enseñanza del electromagnetismo. No pretendemos que sea definitiva. Esperamos que sea sometida a un análisis crítico y dé lugar a una reflexión sobre la metodología que se sigue en las aulas universitarias.

\section{PLANIFICACIÓN CURRICULAR}

En ta planificación curricular tenemos en cuenta principalmente tres factores: la problemática cientifica que se deriva de la temática a estudiar (los contenidos del tema), las ideas o concepciones de los alumnos a los que va dirigida la enseñanza y, sobre todo, las estrategias y proceso metodológico que seguimos. Tampoco olvidamos otros factores con más dificultad de influencia, 
como el nivel de desarrollo cognitivo de los alumnos, sus destrezas y actitudes ante la ciencia, sus intereses, el clima de aula, los recursos con los que contamos, etc.

En el análisis estructural de los contenidos y hechos científicos, utilizamos mapas conceptuales del tipo Novak y Gowin (1984) (Novak 1990) para representar las relaciones significativas entre conceptos y explicitar nuestro pensamiento sobre la temática a impartir. Esta técnica nos ayuda a reflexionar sobre nuestras propias concepciones y puntos de vista en relación con el modo de desarrollar el tema en el aula. Consideramos también el proceso histórico de cómo han evolucionado las ideas cientificas.

Naestra concepción del aprendizaje se sitúa dentro de las teorías del constructivismo. Entendemos el aprendizaje como cambio conceptual (Posner et al. 1982), metodológico (Gil y Carrascosa 1985) y actitudinal. Entre las características del aprendizaje significativo, señaladas por Driver (1986), destacamos de modo especial Ias siguientes:

- Tener en cuenta los conocimientos previos, concepciones y motivaciones de los alumnos, además de crear un entorno adecuado y buenas experiencias de aprendizaje.

- Favorecer procesos que ayuden a los alumnos a ser responsables de su propio aprendizaje. Éste tiene lugar cuando construyen de forma activa sus propios conocimientos.

- Presentar diversas estrategias didácticas dentro de un flexible programa de actividades. Es necesario disponer de un banco de actividades y recursos para ser utilizado según diferentes situaciones, a fin de no dar lugar a la improvisación.

- Considerar que todos los trabajos que impliquen el tratamiento de situaciones problemáticas (trabajos práciicos, resolución de problemas, etc.) son actividades que contribuyen en gran medida a que el aprendizaje sea significativo.

- Potenciar el diálogo y la discusión en el aula, ya que el conocimiento es construido por las personas a través de la interacción social.

Conseguir estos objetivos no es fácil, por lo que nos vemos obligados a considerar el currículo en una óptica de continuo desarrollo. Tiene la naturaleza de instrumento en construcción y adecuación permanente a la realidad educativa y, por tanto, está sometido continuamente a evaluación.

\section{Propuesta metodológica}

Este tercer pilar en que se basa ta planificación de la acción didáctica, lo consideramos de suma importancia. Los principios en que se basa la metodología, los objetivos que persigue y su estructuración fueron presentados por Caballero y Meneses (1991) en la XXIII Bienal de Física celebrada en Valladolid. La secuencia de enseñanza de los conceptos, leyes y procedimientos está estructurada en tres fases. A continuación mencionamos algunos aspectos importantes de cada una de las fases.

\section{Fase de planificación}

El punto de partida de este modelo se basa en la constatación de que la adquisición de aprendizajes significativos requiere que los alumnos estến interesados y tengan inquietud por aprender.

Er esta fase, los alumnos explicitan las ideas y modelos explicativos que poseen en relación con el tema de estudio. Es esencial provocar la inquietud por aprender, lo cual implica, que el alumno, en cierto modo se compromete a participar activamente y a profundizar sobre él mismo. Además de motivarles, la afloración de las ideas constituye un buen instrumento de diagnóstico para el profesor, que le permite elegir y orientar situaciones problemáticas.

Las situaciones problemáticas que se presentan a los alumnos deben poner en duda sus ideas, utilizando contraejemplos y generando conflictos cognitivos. Asimismo deben ser motivantes, relacionadas con el entorno más próximo y adaptadas a la capacidad y conocimientos del alumno. De este modo podrán formular sus propias hipótesis y propuestas de resolución.

\section{Fase de construcción}

Para facilitar la formulación de hipótesis se fomenta la discusión e intercambio de ideas. Aunque en un principio puedan ser ambiguas e imprecisas, sirven de hilo conductor para interpretar observaciones y experimentos. Sin ellas, la utilización de los instrumentos de investigación resulta carente de interés intelectual para el alumno y favorece su utilización mecánica. La formulación de hipótesis es el elemento básico que facilita la construcción de ideas y conceptos interpretativos, ya que, a través de los instrumentos de investigación, los alumnos objetivan lo que piensan.

Éstà es la fase más compleja, pues los estudiantes deben comprobar la coherencia de sus hipótesis. Muchas veces requieren nucvos conceptos para interpretar la situación planteada. Un agente externo les proporcionará nuevos términos y definiciones, bien mediante presentación explícita del profesoro a través de materiales de instrucción. Éstos deben estar expertamente planificados por el profesor o un equipo de investígación. La eficacia o idoneidad de las actividades de aprendizaje que se seleccionen, constituye un problema empírico y su evaluación requicre investigación. Por tanto, concebimos el aprendizaje del mismo modo que Driver y Oldham (1986) como el programa de actividades a través de las cuales dichos conocimientos y habilidades pueden ser construidos y adquiridos.

En la elaboración de los materiales de instrucción es indispensable establecer una secuencia ciara de las actividades a partir de la realidad que ofrecen los centros 
universitarios. Por desgracia, debido a la masificación de la gran mayoría de éstos y a la rigidez de su organización, no se pueden utilizar todos los métodos, recursos y técnicas de investigación que se deseen.

Al final de esta fase, el alumno debe llegar a conceptualizar y estructurar los conocimientos implicados en la investigación. Hasta llegar a una conclusión clara se cometen muchos errores. Tomar conciencia de elloes un clemento básico para avanzar más eficazmente en posteriores investigaciones.

El aprendizaje mediante este método se basa en el ensayo y error progresivo. Permite la construcción de verdades aproximativas, que adquicren siempre una validez relativa. Los errores, con su consiguiente reelaboración, son fundamentales para la maduración de las estructuras intelectuales y la formulación de conceptos. Ei resultado de esta fase será, por tanto, que los alumnos construyan nuevos conceptos, principios o procedimientos.

\section{Fase de ampliación y aplicación}

En esta fase se plantean nuevos problemas para así favorecer y extender el significado del concepto. Se da a los estudiantes oportunidades para usar las nuevas ideas de varias formas y adquirir confianza en las mismas. En algunas situaciones problemáticas se requerirá nueva información, reiniciándose otra vez el ciclo de aprendizaje.

También se diseñan actividades que muestren la utilidad de los principios físicos y contribuyan a que los alumnos vean la relevancia y utilidad de lo aprendido.

Al final del proceso se hace reflexionar a los alumnos sobre el cambio o evolución en sus ideas, realizando comparaciones entre su pensamiento actual y el inicial. Conviene, por tanto, recoger las ideas primitivas para así poder observar la cvolución de las mismas a partir de la instrucción. La utilización de diagramas conceptuales (Novak y Gowin 1988), antes y después de la temática a tratar, es una buena técrica para que el alumno pueda reflexionar sobre su grado de aprendizaje.

El ciclo puede ser utilizado con diversos grados de estructuración y directividad por parte del profesor. La interacción entre alumno-alumno y alumno-profesor son de suma importancia en el proceso de aprendizaje. Así en muchos momentos del proceso proponemos el trabajo en grupos de cuatro alumnos en los que se favorece el diálogo y el acuerdo sobre puntos de vista conflictivos. Estos trabajos en pequeños grupos se alternan con sesiones en el aula, donde cada grupo explica los procedimientos aplicados para resolver las tareas.

En este contex to, cuando los estudiantes intentan «comprenderm los procesos de resolución de problemas desarrollados por otros, amplían su marco conceptual al construir un dominio consensuado con su compañero. Esta característica del diseño instruccional se apoya en la idea de que el lenguaje puede ayudar a reflexionar al estudiante sobre su propia comprensión cuando da expli- caciones, ayudándole a reconceptualizar sus propias construcciones cognitivas (Perret-Clermon 1984),

En el diagrama de la página siguiente hemos representado la sintaxis de las fases.

\section{SECUENCIA DE ENSEÑANZA}

\section{Mapa conceptual}

Para la planificación de la secuencia de enseñanza hemos partido de mapas conceptuales que se han elaborado después de una selección de los conceptos a tratar. Éstos Ios hemos elegido considerando su frecuencia en los contenidos de una muestra de quince libros sobre física general, utilizados babitualmente en el primer curso de estudios universitarios. Los mapas conceptuales pretenden reflejar cómo entendemos la estructura de la disciplina y son una ayuda para desarrollar posteriormente la programación de la instrucción.

La claboración de un mapa conceptual permite profundizar en el conocimiento de la materia y sobre todo cuestionar nuestro propio pensamiento «espontáneo», adquirido a través de lo que hemos visto hacer a nuestros profesores o refiejado en los libros de texto.

En el anexo I presentamos el mapa conceptual global del tema. En él se muestra la mayoría de los conceptos que intervienen en la programación, así como las proposiciones que los relacionan.

\section{Concepciones sobre el electromagnetismo}

Para averiguar las concepciones de los alumnos sobre el electromagnetismo hemos consultado los trabajos publicados (Dupin y Johsua 1986, Furió y Guisasola 1993, Tiberghien y Delacote 1976, Shipstone 1985, Joshua 1984, Psillos et al. 1988). Casi todos se refieren a conceptos básicos de la electrocinética en circuitos de corriente continua. Por tanto, sin apenas puntos de referenciá, preparamos una experiencia piloto en forma de consulta abierta. Pretende identificar algunas ideas o concepciones sobre la relación entre la electricidad y el magnetismo que subyacen en la mente de los alumnos al acceder a la instrucción superior, así como su grado de interiorización.

El anexo Il recoge las seis preguntas abiertas formuladas a los alumnos sobre propiedades de los imanes, origen del campo magnćtico, interacciones entre corriente eléctrica y campo magnético, imantación y aplícaciones del electromagnetismo. Las respuestas anafizadas son 78 y proceden de alumnos del primer curso de la Sección de Qú́micas del Colcgio Universitario de Burgos.

A pesar de las dificultades de clasificación y análisis de las respuestas, dada la naturaleza abierta de la consulta $y$, por tanto, el espectro amplio de respuestas, se detectan una serie de características comunes, con mayor o menor 

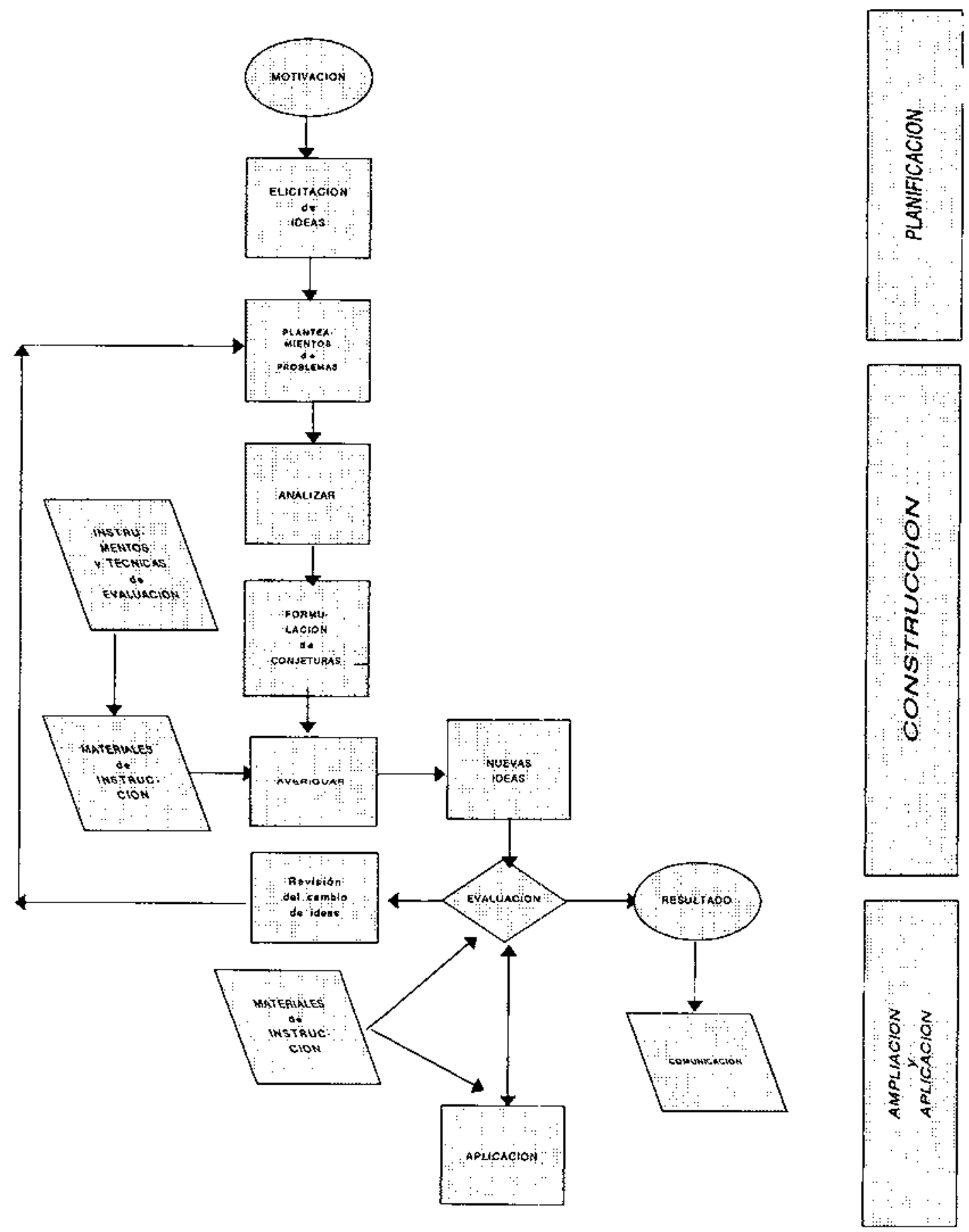

intensidad, a tener en cuenta en la instrucción didáctica de la unidad temática.

Las principales conclusiones extraídas de las respuestas pueden resumirse como sigue:

- Se considera que un imán es una especie de gran dipolo, en cuyo polo norte se encuentran concentradas (o existe un exceso) las cargas positivas y en el polo sur, las negativas. Por ejemplo, responden que, si se acerca un imán recto por uno de sus extremos a un péndulo cargado, lo atraerá o lo repelerá dependiendo del tipo de cargá (positiva o negativa) que tenga la bolita del péndulo y del extremo (norte o sur) del imán más próximo al péndulo.

- Existe una gran confusión entre campo eléctrico y campo magnético. Los alumnos expresan, en general, que un imán crea a su alrededor un campo magnético. Sin 
embargo al predecir sus efectos indican los debidos a la existencia de un campo eléctrico y no magnético. Por ejemplo, señalan con mucha frecuencia que el polo norte de un imán atrae a una carga o cuerpo (en reposo) cargado negativamente y repele a otro cargado positivamente. Asimismo, al explicar la atracción del hierro por un imán, consideran que existe una inducción eléctrica en lugar de magnética; interpretan que en el hierro se produce una redistribución de cargas y mayoritariamente colocan las negativas lo más próximas posible al polo norte del imán. En consecuencia, se desconoce el fenómeno de inducción magnética.

Al explicar los efectos de un imán sobre cuerpos cargados o viceversa casi nunca mencionan el estado de reposo o movimiento de las cargas. Conocen el efecto de una corriente eléctrica sobre una brújula pero no mencionan la existencia de cargas en movimiento. A veces se señala que el efecto de un imán sobre una carga consiste en ponerla en movimiento.

- Se cree que las interacciones visibles se producen entre imanes y toda sustancia metálica. Desconocen, en su mayoría, la existencia de sustancias diamagnéticas, paramagnéticas y ferromagnéticas y su interacción (aunque sea débilmente) con un imán. Se hace escasa referencia a los conceptos de carga eléctrica en movimiento $e$ intensidad de corriente (ésta algo más que la carga) como fuentes del campo magnético. Prácticamente se ignoran los efectos que produce un campo magnético sobre una carga elćctrica en movimiento o sobre una intensidad de corriente eléctrica. A esta misma conclusión también llegan otros autores (Pontes et al. 1991). Esto demuestra un deficiente nivel de aprendizaje sobre el origen del magnetismo, detectado también en otras investigaciones (Colombo 1990).

Para explicar la interacción entre imanes y metales, los alumnos dan varias explicaciones:

a) Asocian al metal la doble polaridad del imán sin dar detalles.

b) Creen que el imán tiene en sí mismo una fuerza capaz de atraer cuerpos ("efecto mágico»).

c) Estiman que hay un paso (transferencia) de carga del imán al hierro, produciéndose la interacción al estar los dos cargados. Esta concepción de la carga como un fluido que fluye con facilidad se la ha llamado «sustancialista" (Guisasola y Furió 1991), y es un preconcepto cuyas raíces podráan estar en los modelos mecanicistas que proponfan los científicos de la primera mitad del siglo xvill.

d) Consideran, como ya se ha dicho, que se produce una redistribución de cargas en el hierro (orientación de los dipolos eléctricos), en lugar de la orientación de los dipolos magnéticos que son totalmente desconocidos.

- Hay un cierto conocimiento de experiencias sobre fenómenos electromagnéticos. Los más conocidos son: el experimento de Oersted (15\%), las experiencias sen- cillas de inducción eléctrica -mover un imán en las proximidades de un circuito- $(25 \%)$ y el electroimán $(20 \%)$.

Estas experiencias nos sirven como punto de partida en la secuencia didáctica. Los alumnos al explicar los fenómenos que intervienen en ellas explicitan sus ideas. En consecuencia, se utilizan para introducir los conceptos de campo magnético, inducción electromagnética, ferromagnetismo, imantación, etc.

- Un porcentaje elevado (30\%) no menciona (desconoce) experiencias o fenómenos que se relacionen con estos hechos. Consideramos muy preocupante que un alumno, ya universitario, sea incapaz de realizar un posible diseño sobre cualquier cuestión que se Ie proponga. Este hecho pone en evidencia la escasa importancia que se atribuye a este aspecto clave en toda investigación científica. No se pretende que describan correctamente lo que ocurre en los experimentos sino que confeccionen diseños sencillos y susceptibles de ser realizados en el aula o laboratorio. Uno de nuestros objetivos de la asignatura consiste precisamente en potenciar una verdadera aplicación de la metodología científica, en donde aspectos claves y escasamente tenidos en cuenta como la emisión de hipótesis, planificación de diseños experimentales, confección de informes, etc. sean considerados en su justa medida.

- La mayoría de los alumnos conocen que la Tierra es un gran imán, pero desconocen que cerca del polo norte geográfico se encuentra el sur magnético y que cerca del polo sur geográfico se encuentra el norte magnético.

Para explicar que una aguja magnética se orienta hacia el norte geográfico dan las siguientes razones:

- Existe un potente imán en el norte geográfico capaz de atraer la aguja metálica de la brứjula, aunque se encuentre muy lejos.

- Debajo de la aguja de la brújula existe un imán que es orientado por el imán Tierra. Es curiosa esta respuesta, pues, a pesar de que en el enunciado de la cuestión se especifica que se trata de una aguja magnética, no parece creerlocl alumno. Se intuye, de esta respuesta, la dificultad que encuentran para comprender que una aguja pueda ser un imán (pueda estar imanada), quizás debido a su forma delgada o a su poco peso.

- Al explicar cómo está constituido un electroimán, indican que se trata de un imán cargado al que se le ha enrrollado un conductor por el que circula una corriente. No parecen darse cuenta de que el núcleo del electroimán se imanta y desimanta con facilidad; creen que se trata de un imán permanente. Saben que la corriente eléctrica es fundamental para el funcionamiento, pero no comprenden fácilmente que su función es imantar el núcleo.

En ningún caso se menciona la palabra imantación (o similar) del núcleo debido a la corriente y, sin embargo, se señala frecuentemente que el núcieo se carga y descar- 
ga al aplicar o quitar respectivamente la corriente. A veces mencionan que la corriente induce un imán.

- Se constata un desconocimiento casi total del efecto que produce un imán sobre una corriente. Sin embargo, el efecto contrario es relativamente conocido. Parece aconsejable, por tanto, introducir primero el efecto que produce una corriente eléctrica sobre un imán y posteriormente el efecto que produce un imán sobre una corriente o carga en movimiento.

\section{Programa de actividades}

Mediante el conjunto de actividades que hemos confeccionado, pretendemos que los alumnos lleguen a superar las concepciones incorrectas. Nuestra hipótesis señala que esto se consigue con la metodología que hemos desarrollado anteriormente

El anexo III muestra actividades propuestas sobre propiedades magnéticas de la materia. Por falta de espacio no presentamos la unidad didáctica completa en la que se incluye este apartado. La secuencia de actividades no es rigida, la variamos en función de los comportamientos e intereses de los alumnos.

La unidad contiene 71 actividades y está estructurada en diez apartados:

I. Fenómenos magnéticos: problemas que plantean.

2. Relación entre la electricidad y el magnetismo.

3. Acción del campo magnético sobre cargas en movimiento.

4. Campo magnético creado por cargas en movimiento.

5. Teorema de Ampère: aplicaciones.

6. Interacciones entre corrientes eléctricas.

7. Propiedades magnéticas de la materia.

8. Aplicaciones del electromagnetismo.

9. Flujo y circuitos magnéticos.

10. Inducción electromagnética: leyes de Faraday y Lenz.

Las actividades que figuran en la unidad son muy diversas. Se proponen actividades para explorar y discutir las ideas previas de los alumnos (A.32), para provocar el conflicto conceptual (A.33 y A.34), para introducir modelos y teorías científicas (A.40), para aplicar las nuevas ideas a distintos contextos y a la resolución de problemas (A. 42 y A.43), para comparar las idcas antiguas con las nuevas (A.40), etc. En definitiva la unidad comprende tres grandes bloques de actividades: de planificación y exploración, de construcción y de ampliación y aplicaciốn.
Algunas actividades proponen la formulación de hipótesis (A.7), el diseño (A.8) y la realización de experimentos (A.9), la contrastación de hipótesis (A.13), la formulación de conclusiones (A.19), eic. De este modo, el alumno puede potenciar habilidades relacionadas con los procesos de la ciencia, al actuar como un «pequeño» investigador intentando dar solución a situaciones problemáticas más o menos abiertas.

En otras actividades, por la dificultad que entraña la temática a tratar, no se podrán plantear situaciones experimentales. En estos casos se recurre a la presentación, por parte del profesor, de «ayudas» (textos históri$c o s$, datos experimentales simulados, conceptos nuevos, etc.) que hagan reflexionar al alumno sobre sus propias ideas, manteniéndole activo. Sólo podrá reestructurarlas si está implicado en su propio aprendizaje.

\section{CONSIDERACIONES FINALES}

Mediante la secuencia de actividades que hemos diseñado no sólo pretendemos que los alumnos aprendan significativamente los principales conceptos electromagnéti$\cos$, sino también que se vayan acostumbrando a otra forma de trabajo, en donde el auténtico protagonista sea tuno mismo.

La secuencia presentada no debe considerarse como absolutamente rígida; en función de las características de los alumnos y de cómo vayan estableciendo las conclusiones, el profesor podrá modificar los pasos a seguir, sin olvidar en ningún instante, las metas que pretende alcanzar. Se requiere, por tanto, disponer de un amplio «banco» de actividades para no dar lugar a la improvisación.

Consideramos muy importante explicar al alumno que el cambio de metodología supone una modificación sustancial en la evaluación. No sólo debemos valorar el aprendizaje de conceptos y estructuras conceptuales, sino también el aprendizaje de procedimientos, la adquisición de habilidades y estrategias en la resolución de problemas, la competencia en el uso de los métodos de la ciencia, la actitud hacia la materia, etc. Sólo si el alumno observa y se compromete a este sistema de evaluación, modificará su conducta de actuación y podrá producirse el cambio metodológico.

Este nuevo enfoque requiere un entrenamiento, tanto por parte del profesor como de los alumnos. I Hemos detectado que los profesores valoran positivamente el método de trabajo que se sigue en el aula y se entusiasman por el mismo rápidamente. Toman conciencia de que enseñar no es tan fácil y requiere un conocimiento profundo de la materia, un cuestionamiento continuo del modo de enseñar y conocimientos teórico-prácticos sobre el proceso de enseñanza-aprendizaje de las materias científicas. Los alumnos, por el contrario, necesttan más ticmpo para adaptarse a esta manera de trabajar. También valoran positivamente la metodología, aunque ello les exija más dedicación. 


\section{REFERENCIAS BIBLIOGRÁFICAS}

BARNES, D., 1969. From Communication to Curriculum. (Penguin Books: Hammonds Woth, Inglaterra).

CABALLERO, C. y MENESES, J.A., 1991. Una propuesta metodológica para la enseñanza de la física a nivel universitario, XXIII Reunion Bienal de Física. (RSEF: Valladolid).

COLOMBO DE CUDMANI, L. y FONTDEVILLA, P.A., 1990. Concepciones en el aprendizaje significativo del electromagnetismo, Enseñanza de las Ciencias, 8(3), pp. 215-222.

DRIVER,R., 1986. Psicología cognoscitiva y esquemas conceptuales de los alumnos, Enseñanza de las ciencias, 4(1), pp. 3-15.

DUPIN, J.J. y JOSHUA, S., 1986. Evolución de las representaciones de los alumnos y el impacto de la enseñanza sobre sus representaciones, Bulletin de l'union des physiciens, 683 , pp. $779-800$.

ERICKSON, G.L., 1979. Children's conceptions of heat and temperature, Science Education, 63, pp. 221-230.

FURIÓ, C. y GUISASOLA, I., 1993. ¿Puede ayudar la historia de la ciencia a entender por qué los estudiantes no comprenden los conceptos de carga y potencial eléctricos?, Revista Española de Fisica, 7(3).

GIL,D. y CARRASCOSA, J., 1985. Science learnig as a conceptual and methodological change, European Journat of Science Education, 7(3), pp. 231-236.

GUISASOLA, J. y FURIÓ, C., 1991, Dificultadesenel aprendizaje significativo de algunos conceptos de electrostática, Actas de la XXIII Reunión Bienal de la RSEF.

IOSHUA, J., 1984. Student's interpretation of simple electrical diagrams, European Journal of Science Education, 6, pp. $271-275$.

KARPLUS, R., 1977. Science Theaching and the Development of Reasoning. (University of California: Berkeley, California).

NEEDHAM, R., 1987. Teaching strategies for development understanding in Science, Children's learning in Science Projet, University of Leeds.
NOVAK, J.D., 1990. Concept mapping: a useful tool for science educatión, Journal of Research in Science Teaching, 27(10), pp. 937-949.

NOVAK, J.D. y GOWIN, D., 1984. Learning How to Learn (Cambridge University Press: Cambridge).

NUSSBAUM, J. y NOVICK, S, 198I. Brain storming in the classroom to invent a model: a case study, School Science Review, 62, 221, pp. 771-778.

OSBORNE, R.I. y WITTROCK, M.C., 1983. Learning science: A generative process, Science Edacation, 67(4), pp. $489-508$.

PERRER-CLERMONT, A., 1984. La construcción de la inteligencia en la interacción social. (Visor: Madrid).

PONTES, A., GAMERO, A. y ROSADO, L., 1991, Una investigación sobre los preconceptos del electromagnetismo al inicio de la enseñanza universitaria, Actas de la XXIII Reunión Bienal de la RSEF, pp. 62-63.

POSNER, G.J., STRIKE,K.A., HEWSON, P.W, y GERTZOG,W.A., 1982. Accomodation of a scientific conception: towards a theory of conceptual change, Science Education, 66(2), pp. 211-227

PSILLOS et al., 1988. Voltage presented as a primary concept in an introductory teaching sequence on DC circuits, International Journal of Science Education, 10(1), pp. 2943.

SHIPSTONE, D., 1985. Children's Ideas In Science, cap. 8, Electricity in Simple Circuits, pp. 33. R. Driver. Open University Press.

RENNER, J., 1982. The power of purpose, Science Education, 66(5), pp. 709-716.

ROWEL y DAWSON, 1983. Laboratory counter examples and the growth of understanding in Science, European Journal of Science Education, 5(2), pp. 203-2 I5.

TIBERGHIEN, A. y DELACOTE, G., 1976. Manipulations et representations de circuits électriques simples par des enfants de 7 a 12 ans, Revue Française de Pedagogie, 34, pp. 32-44. 


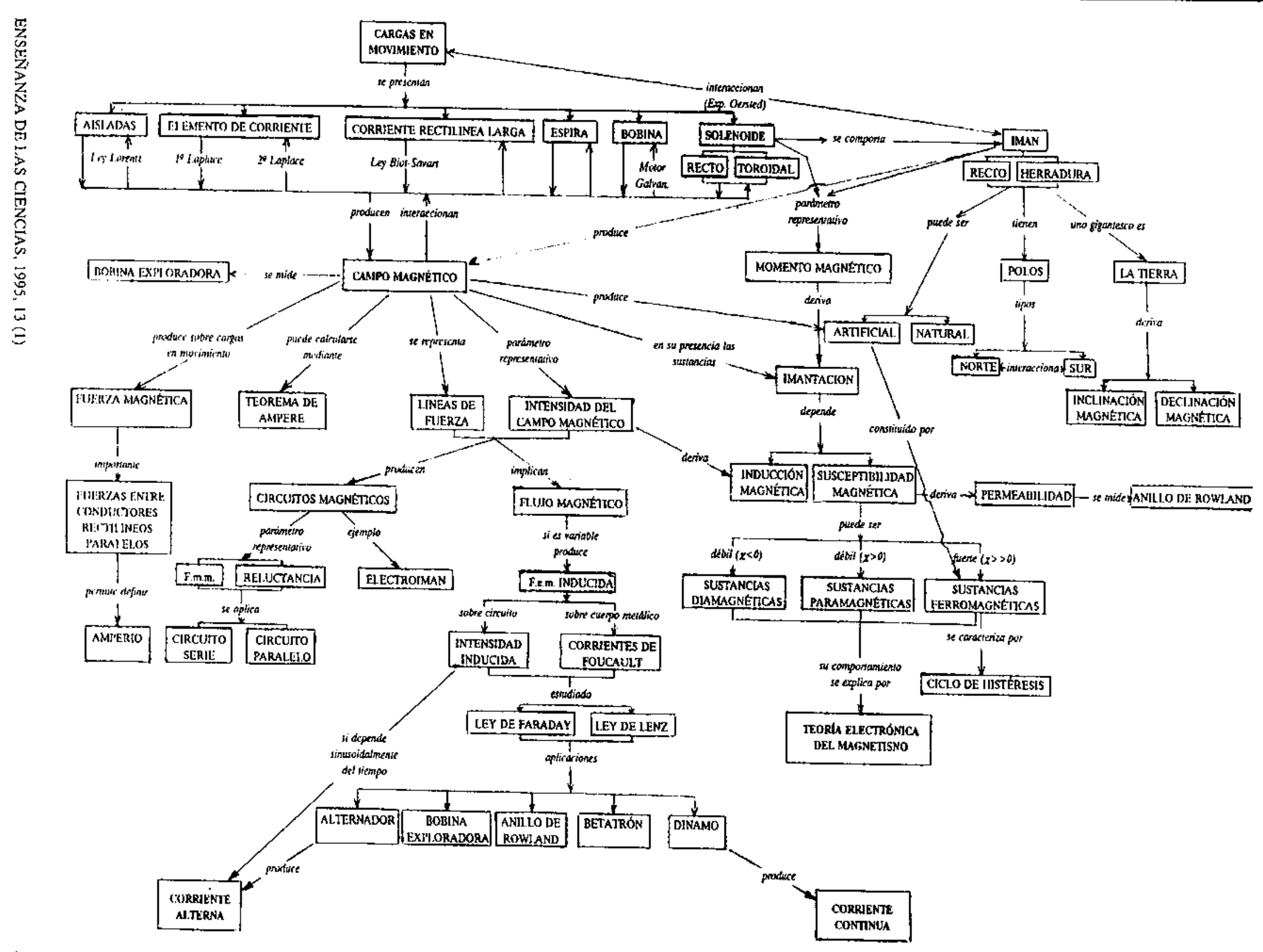




\section{ANEXO 2}

1) Indica las propiedades que conozcas de los imanes.

2) ¿Qué experiencia diseñarías para demostrar la relación entre los fenómenos eléctricos y magnéticos?

3) ¿Cómo explicas el hecho de que una aguja magnética (brújula) señala siempre hacia el norte?

4) En la figura se representa un péndulo y un imán. Explica lo que ocurre sobre la bola del péndulo.

a) Si la bola es de madera cargada positivamente.

b) Si la bola es de hierro y está en estado neutro.

5) Para cargar chatarra en un camión se suele utilizar una grúa que dispone de un electroimán.

a) Explica qué es un electroimán (su constitución) y por qué atrae a la chatarra.

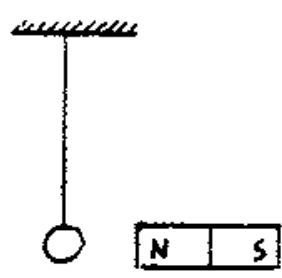

b) Explica cómo se puede separar la chatarra del electroimán para depositarla en el camión.

6) Si acercamos un imán a un conductor rectilíneo por el que circula una corriente, ¿se produce algún efecto?

\section{ANEXO 3}

\section{PROPIEDADES MAGNÉTICAS DE LA MATERIA}

Una vez establecido que los campos magnéticos son creados por cargas en movimiento, fue posible abordar el problema de la naturaleza del magnetismo natural.

Ampère descubrió que una pequeña espira de corriente experimenta el mismo par de fuerzas en un campo magnético y da origen al mismo campo magnético que si fuese un dipolo magnético. En 1820 , basado en esta observación, enunció el teorema de la equivalencia de Ampère:

«Una espira de corriente es equivalente en todas sus propiedades magnéticas a un dipolo magnético a distancias grandes comparadas con las dimensiones de la espira».

Esta equivalencia lievó a Ampère a creer que los polos magnéticos se deben realmente a las corrientes que circulan en el interior de la materia. Así, el campo de un imán en forma de barra es el campo neto producido por muchas espiras moleculares de corriente.

A.32. Propon un modelo (teoría) que explique el magnetismo natural.

A.33. Contesta vuestro modelo a hechos como los siguientes:

a) No se pueden separar los polos de un imán.

b) Un imán no atrae a todas las sustancias

c) Al situar un núcleo de hierro o acero en las proximidades de un imán, se convierte a su vez en un imán. Compruébalo.

d) Frotando una aguja de acero con un imán, siempre en la misma dirección y con el mismo polo, desde el centro a uno de los extremos, se convierte en un imán. Experiméntalo.

e) Arrolkando un cable, por el que circula una corriente continua, a una aguja de acero, ésta se convierte en un imán. Sin embargo, si la corriente es alterna, no se consigue imantar la agtuja. Compruébalo.

f) Se puede conseguir un imán potente utilizando varios débiles. ¿Cómo lo harías?

A.34. Representa las líneas de fuerza del campo magnético producido por el imán de la figura.

Coloca trozos de hierro, cobre y aluminio en el entrehierro del imán de herradura de la figura y visualiza las líneas de fuerza.

Explica por qué al colocar un tipo de stistancias en la posición A se desplazan hacia arriba; sin embargo al colocar otro tipo se desplazan hacia abajo.

¿Explica vuestro modelo los fenómenos anteriores? Modifícalo si es preciso. 
A.35. Coloca un trocito metálico proveniente de los electrodos de una vieja válvula electrónica rota (lleva níquel) colgado a modo de péndulo mediante un alambre fino. Acerca un imán de modo que «el péndulo» quede desviado de la vertical por la atracción del imán. Acerca ahora con cuidado lá llama de un mechero de modo que caliente el metal y no el imán. ¿Qué sucede al cabo de unos segundos? ¿A qué se debe? Espera a que se cnfríe el metal y, si el imán está suficientemente cerca, ¿qué vuelve a ocurrir?, ¿por qué?

Iustifica el modelo o teoría este fenómeno. Modifícalo en caso contrario.

Como ya sabes, al introducir cualquier sustancia dieléctrica en un campo eléctrico, éste queda modificado (dísminuye), ya que se polariza dicha sustancia. Del mismo modo, un campo magnético queda alterado (aumenta o disminuye) cuando colocamos en él cualquier sustancia; ésta se imana o imanta.

En un principio se creía que sólo se producía un fenómeno de atracción al colocar algunas sustancias, como el hierro, cerca de un imán. Sin embargo A.J. Brugmans descubrió en 1778, experimentando con el antimonio y el bismuto, que eran repelidos al acercalos a un imán. En los años que siguicron a 1845, Faraday investigó numerosas sustancias y acuno los términos diamagnetismo y paramagnetismo para describir aquellas sustancias que se ven repelidas o atrafdas respectivamente por un imán permanente. Descubrí que muchos elementos y la mayoría de los compuestos eran diamagnéticos.

A.36. Compara los efectos mencionados, estableciendo las semejanzas y diferencias.

Defíne la imantación o imantación de manera análoga a la polarización. ¿De qué depende?

A.37. Calcula la imantación que existe en el interior de un solenoide recto por el que circula una corriente en función del campo magnético que crea.

Indicar un procedimiento para aumentar la intensidad del campo magnético existente en una zona del espacio; por ejemplo, en el interior de la bobina anterior. Compruébalo. ¿En cuánto aumentaría la intensidad del campo?

A.38. Indica cómo se puede saber la imantación y el campo magnético que existe en la barra de hierro de la figura adjunta.

A.39. Comentario del profesor sobre diferentes curvas

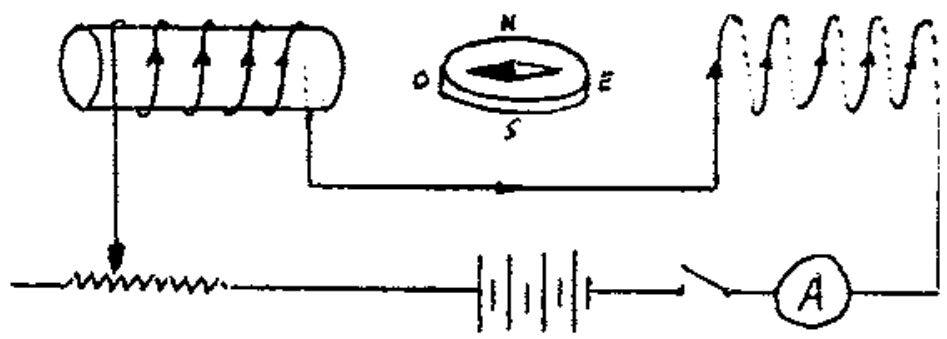
de imantación de materiales ferromagnéticos. Ciclos de histéresis.

A.40. Exposición por el profesor de la teoría electrónica del magnetismo. Interpretación del diamagnetismo, paramagnetismo y ferromagnetismo.

A.41. Coloca una aguja magnética (brújula) de manera que pueda oscilar fibremente en un plano horizontal. Hacer lo mismo de modo que pueda oscilar en un plano vertical. ¿Por qué la aguja señala, en ambos casos, una misma dirección y sentido? ¿Qué dirección y sentido tiene el campo magnético en ese lugar? ¿Dónde consideras que están los polos magnéticos del imán Tierra?

Busca en la bibliografía la representación del campo magnético terrestre e interpreta el significado de inclinación magnética (ángulo que forma el campo con el horizonte) y declinación (ángulo que forma la aguja cuando osciła en el plano horizontal con el meridiano del lugar).

A.42. ¿Cuántas oscilaciones dará un imán en forma de barra cilindrica cuando oscila, suspendido horizontalmente por su centro, en un punto determinado de la Tierra? ¿Cómo puede conocerse el valor del ángulo de inclinación del lugar? ¿Cuál es la imantación y los polos magnéticos provocados en la barra?

A.43. Según el modelo atómico de Bohr, para el hidrógeno se supone que el electrón gira alrededor del núcleo en una orbita circular. ¿Qué fuerza actúa sobre el electron si el átomo está situado en un campo magnético perpendicular a su brbita y sumergido en agua? ¿Cuál es el campo magnético en el núcleo? ¿Cuál es el momento magnético đel átomo? 